\title{
Endoplasmic Reticulum Stress Affects Lipid Metabolism in Atherosclerosis Via CHOP Activation and Over-Expression of miR-33
}

\author{
Yan Sun ${ }^{\mathrm{a}}$ Dai Zhang ${ }^{\mathrm{a}}$ Xiaoli Liu ${ }^{\mathrm{a}}$ Xuesong Lib ${ }^{\mathrm{b}}$ Fang Liu ${ }^{\mathrm{a}} \quad \mathrm{Yi} \mathrm{Yu}^{\mathrm{a}}$ \\ Shuo Jiaa Yujie Zhou ${ }^{\mathrm{a}}$ Yingxin Zhao
}

aDepartment of Cardiology, Beijing Anzhen Hospital, Capital Medical University, Beijing Institute of Heart Lung and Blood Vessel Disease, The Key Laboratory of Remodeling-related Cardiovascular Disease, Ministry of Education, Beijing, 'Key Laboratory of Cardiovascular and Cerebrovascular Medicine, School of Pharmacy, Nanjing Medical University, Nanjing, China

\section{Key Words}

Atherosclerosis $•$ Endoplasmic reticulum stress $\bullet$ Lipid metabolism $\bullet$ miR-33

\begin{abstract}
Background/Aims: Endoplasmic reticulum (ER) stress is an important event in atherosclerosis. Recent studies have shown that ER stress deregulates cholesterol metabolism via multiple pathways. This study aimed to determine the relationship between ER stress and lipid metabolism and to verify that upregulation of miR-33 is involved in this process. Methods: An atherosclerosis model was established in apolipoprotein E-deficient (ApoE $\mathrm{E}^{-/-}$) mice fed a Western diet, and THP-1 derived macrophages were used in this study. Hematoxylin-eosin and Oil Red $\mathrm{O}$ staining were used to quantify the atherosclerotic plaques. 1,1'-Dioctadecyl3,3,3',3'-tetramethylindocarbocyanine perchlorate labeled oxidized low-density lipoprotein binding assay and a Cholesterol Efflux Fluorometric Assay Kit were used to observe cholesterol uptake and efflux. The mRNA and protein levels of biomarkers associated with ER stress and cholesterol metabolism in atherosclerotic plaques and macrophages were evaluated by real-time PCR and western blotting, respectively. Immunofluorescence was used to observe alterations of ABCA1 localization. Small interfering RNAs were used to knock down CHOP and miR-33 in macrophages to alter CHOP and miR-33 expression. Results: Atherosclerotic lesions and systemic lipid levels were ameliorated after inhibition of ER stress (tauroursodeoxycholic acid) in vivo. In vitro studies confirmed that ER stress regulated the lipid catabolism of macrophages by promoting cholesterol uptake, inhibiting cholesterol efflux, and modulating the expression of related transporters. CHOP contributed to lipid metabolism disorder following ER stress. Furthermore, over-expression of miR-33 was involved in ER stress that induced lipid metabolism disorder in macrophages. These findings support a model of ER
\end{abstract}

Yan Sun and Dai Zhang contributed equally to this work.

\footnotetext{
Yujie Zhou Dept. of Cardiology, Beijing Anzhen Hospital, Capital Med. Univ., Beijing Inst. of Heart Lung and Blood Vessel Disease and Xiaoli Liu No. 2 Anzhen Road, Chaoyang District, Beijing 100029, (China) Tel. 86-10-64456489, E-Mail azzyj12@163.com; liux19881@163.com
} 
stress induction by oxidized low-density lipoprotein that affects macrophage lipid catabolism disorder. Conclusion: Our data shed new light on the relationship between ER stress and lipid metabolism in vivo and in vitro, and confirm that upregulation of miR-33 is involved in this process. The relationship between ER stress and miR-33 represents a novel target for the treatment of atherosclerosis.

\section{Introduction}

Atherosclerosis is a chronic inflammatory disease characterized by the accumulation of cholesterol-laden macrophages, cell death, and fibrosis in artery walls [1]. Maintenance of intracellular lipid homeostasis is vital for cell survival and function, and macrophages play a pivotal role in atherogenesis through the regulation of lipid metabolism $[2,3]$. Normally, modified low-density lipoproteins (LDLs) are engulfed by scavenger receptors (SRs) on macrophages and removed through reverse cholesterol transport [4, 5]. Then, lipiddepleted macrophages can migrate from the intima to reduce lesional inflammation. When lipids are present in excess, the metabolic capacity of macrophages is impaired, accelerating macrophage foam cell formation and contributing to plaque formation, plaque rupture, and acute cardiac death $[3,6,7]$. Therefore, efficient removal of lipids is essential for the prevention of foam cell formation and for the reversal of lipid buildup in atherosclerotic plaques. Treatments directed toward lipid removal represent a promising strategy for the treatment of atherosclerosis [8].

The endoplasmic reticulum (ER) is responsible for several functions that are necessary to maintain cellular homeostasis such as folding and transport of secretory and membrane proteins [9]. However, the accumulation of misfolded proteins (ER stress) triggers the unfolded protein response (UPR), which is an important event during the initiation, development, and clinical progression of atherosclerosis $[10,11]$. High concentrations of stressors such as intracellular free cholesterol, oxysterols, and oxidized LDL (ox-LDL) can provoke ER stress severe enough to activate lipid-laden macrophage apoptosis and plaque necrosis [12]. However, in some circumstances, ER stress alone does not induce apoptosis in macrophages [12-15]. The role of ER stress in atherosclerosis is not limited to the activation of apoptotic responses, as it also participates in macrophage lipid metabolism, inflammation, and metabolism. A recent study showed that ER stress deregulates cholesterol metabolism in human hepatic cells by inhibiting 3-hydroxy-3-methyl glutaryl coenzyme A reductase-mediated cholesterol synthesis and by impairing ATP-binding membrane cassette transport protein A1 (ABCA1)-mediated cholesterol efflux. These findings suggest a connection between lipid metabolism and ER stress [16]. However, the signaling networks linking macrophage ER function, lipid metabolism, and atherogenesis remain obscure. Here, we investigated the mechanisms that contribute to cholesterol metabolism disorder in macrophages, with a focus on ER stress.

CCAAT/enhancer-binding protein-homologous protein (CHOP) is a leucine zipper transcription factor present at low levels under normal conditions, but its expression is enhanced in response to ER stress [17]. CHOP is significantly increased in macrophages from thin-cap atheroma and ruptured plaques, which represent evidence of ER stress and UPR activation at advanced stages in atherosclerosis [18]. ER stress-induced apoptosis associated with the upregulation of CHOP has been demonstrated in atherosclerotic lesions in humans and apolipoprotein E-deficient (ApoE $\left.{ }^{-/}\right)$mice. Moreover, several studies have observed the same tendency in CHOP levels and cholesterol accumulation in atherosclerotic models, although the relationship between CHOP and cholesterol metabolism remains inconclusive $[19,20]$. Thus, in addition to its role as a key signaling component of ER stressinduced apoptosis during atherosclerosis, the role of CHOP in lipid metabolism has yet to be explored.

Intracellular levels of cholesterol are tightly regulated via microRNAs (miRNAs) [21]. miRNAs are highly conserved noncoding RNAs that act as post-transcriptional inhibitors of 
gene expression [22]. One of the best-characterized miRNAs regulating cholesterol efflux is miR-33, which is an intronic miRNA located within the sterol regulatory element-binding transcription factor genes. miR-33 regulates high-density lipoprotein (HDL) biogenesis and cholesterol efflux by downregulating $A B C$ transporter expression in a variety of cell types [23-25]. Anti-miR-33 therapy promotes cholesterol efflux from macrophages, which reduces plaque progression in mouse models of atherosclerosis [26-28]. In human studies, the expression of miR-33 is significantly increased in carotid atherosclerotic plaques. Furthermore, miR-33 expression is markedly increased in hypercholesterolemic patients compared with normocholesterolemic counterparts, thus indicating the important role of miR-33 in regulating cholesterol efflux and atherosclerosis progression [29, 30].

The aim of this study was to verify that CHOP contributes to macrophage lipid metabolism disorder and investigate whether miR-33 is involved in ER stress-mediated inhibition of cholesterol efflux. We inhibited ER stress with tauroursodeoxycholic acid (TUDCA) to study the effects on atherosclerotic lesions and systemic lipid levels in ApoE1- mice. The relationship between ER stress and miR-33 represents a novel target for the treatment of atherosclerosis.

\section{Materials and Methods}

\section{Cell Culture and Reagents}

Human THP-1 monocytes (ATCC, Manassas, VA) were cultured in RPMI 1640 medium containing 10\% fetal bovine serum with penicillin $(20 \mu \mathrm{g} / \mathrm{mL})$ and streptomycin $(20 \mu \mathrm{g} / \mathrm{mL})$ at $37^{\circ} \mathrm{C}$ and $5 \% \mathrm{CO}_{2}$ Cells at logarithmic growth phase were seeded at $10^{6}$ cells $/ \mathrm{mL}$ in $100 \mathrm{ng} / \mathrm{mL}$ phorbol-12-myristate-13-acetate (PMA) for $72 \mathrm{~h}$ and differentiated into adherent THP-1 derived macrophages.

PMA, thapsigargin (TG), tunicamycin (TM), and TUDCA were purchased from Sigma-Aldrich (St. Louis, MO). Antibodies against transcription factor 4 (ATF4), glucose-regulated protein 78 (GRP78), class B SR (CD36), ABCA1, and apolipoprotein A-I (ApoA-I) were purchased from Abcam (Cambridge, UK). ATP-binding cassette transporter G1 (ABCG1) and SR class B type I (SR-BI) were obtained from NOVUS Biologicals (Littleton, CO). CHOP was obtained from Cell Signaling Technology (Beverly, MA), and horseradish peroxidase (HRP)-conjugated secondary antibodies and $\beta$-actin were obtained from ZSGB-BIO (Beijing, China). Fluorescent dyes, including Hoechst 33342 and 4',6-diamidino-2-phenylindole (DAPI), were purchased from Molecular Probes (Invitrogen, Carlsbad, CA). Chemical agents such as PMA, TG, TUDCA, and TM were dissolved in dimethyl sulfoxide $(<0.1 \%)$ prior to use.

\section{Animal experiments}

Male $\mathrm{C} 57 / \mathrm{Bl6J}$ and $\mathrm{ApoE}^{-\%}$ mice (6 weeks old) were purchased from the Animal Centre of Capital Medical University (Beijing, China). Mice were fed a normal diet or a Western diet $(0.25 \%$ cholesterol, $21 \%$ fat, $19.5 \%$ casein, $34.16 \%$ sugar) for 16 weeks. All experimental procedures were approved by the Animal Care and Use Committee of Capital Medical University.

Twenty-four male mice were assigned to the control group (C57/Bl6] mice, $\mathrm{n}=8$ ), model group (ApoE/ mice, $\mathrm{n}=8$ ), and TUDCA group (ApoE $\mathrm{E}^{-/}$mice, $\mathrm{n}=8$ ). Mice received a daily dose $\left(80 \mathrm{mg} \cdot \mathrm{kg}^{-1}\right.$ ) of TUDCA or vehicle during the final 4 weeks.

Blood and aorta samples were harvested for analysis. Blood samples were collected from the angular vein and stored at $-80^{\circ} \mathrm{C}$ until measurement of plasma total cholesterol (TC) and LDL-cholesterol (LDL-C) with a Hitachi 7170 automatic analyzer (Hitachi Limited, Tokyo, Japan). Aortic roots were fixed in 4\% paraformaldehyde, embedded in paraffin, and sliced to 5 - $\mu \mathrm{m}$ thickness for hematoxylin-eosin (H\&E) staining. Aorta samples were dissected and perfused to allow for quantification of atherosclerotic lesions with Oil Red 0 staining, as described previously [31]. Lipid deposits were quantified in a blinded fashion with Image s software (NIH, Bethesda, MD, USA). Remaining aorta samples were stored at $-80^{\circ} \mathrm{C}$. Total protein was harvested for western blot analysis of biomarkers related to ER stress and cholesterol metabolism. Mice were euthanized via pneumothorax after tissue collection.

Immunofluorescence

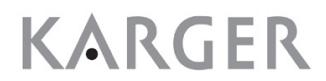




\section{Cellular Physiology Cell Physiol Biochem 2018;48:1995-2010 \begin{tabular}{l|l} 
and Biochemistry Published 10.1159/000492522 & $\begin{array}{l}\text { (c) 2018 The Author(s). Published by S. Karger AG, Basel } \\
\text { www.karger.com/cpb }\end{array}$
\end{tabular} \\ Sun et al.: Endoplasmic Reticulum Stress and Lipid Metabolism}

After treatment, cells were seeded in glass-bottom cell culture dishes, fixed in $4 \%$ paraformaldehyde, and permeabilized with $0.1 \%$ Triton X-100, followed by blocking with $3 \%$ bovine serum albumin (BSA). They were then immunolabeled with specific primary antibodies (ABCA1, 1:100) overnight at $4{ }^{\circ} \mathrm{C}$. After rinsing, the cells were incubated with the corresponding FITC-conjugated secondary antibody (1:250) in $1 \%$ BSA for $1 \mathrm{~h}$ at $37^{\circ} \mathrm{C}$. Nuclei were stained with DAPI for $5 \mathrm{~min}$ at room temperature. Fluorescence was detected by a LEICA TCS-SP2 laser confocal microscope (Leica Microsystems, Wetzlar, Germany).

\section{1, 1'-Dioctadecyl-3, 3,3',3'-tetramethylindocarbocyanine perchlorate labeled ox-LDL binding assay}

Ox-LDL uptake was tracked with the 1, 1'-dioctadecyl-3, 3,3',3'-tetramethylindocarbocyanine perchlorate labeled ox-LDL (DIL-ox-LDL; Peking Union-Biology Co., Ltd., Bejing, China). After treatment with TG or TUDCA for $18 \mathrm{~h}$, macrophages were incubated with DIL-ox-LDL ( $50 \mu \mathrm{g} / \mathrm{mL}$ ) for $6 \mathrm{~h}$ at $37^{\circ} \mathrm{C}$ in the dark. Binding was measured at 488/565 $\mathrm{nm}$.

\section{Oil Red $O$ staining}

Oil Red O (Sigma-Aldrich) was used to measure lipid accumulation and foam cell formation after TG or TUDCA treatment. Differentiated macrophages were cholesterol-loaded with $50 \mu \mathrm{g} / \mathrm{mL}$ ox-LDL for $72 \mathrm{~h}$. Cells were fixed with $4 \%$ paraformaldehyde, followed by dehydration with $60 \%$ isopropanol for 2 min. Neutral lipids were stained with filtered $0.3 \%$ Oil Red 0 solution for 10 min at room temperature. Hematoxylin was used to counterstain cell nuclei for $1 \mathrm{~min}$ after rinsing with $60 \%$ isopropanol. Oil Red 0-stained lipid drops were observed by light microscopy.

\section{Cholesterol efflux}

The capacity of macrophages to degrade cholesterol was measured with the Cholesterol Efflux Fluorometric Assay Kit (BioVision, Inc., Milpitas, CA) according to the manufacturer's instructions. Briefly, macrophages in 96-well plates were incubated with fluorescence-labeled cholesterol for $16 \mathrm{~h}$ at $37^{\circ} \mathrm{C}$. After preincubation with TG or TUDCA, macrophages were treated with cholesterol acceptor HDL (50 $\mu \mathrm{g} / \mathrm{well}$ ). The supernatants and cell monolayers were then solubilized by cell lysis buffer and transferred to a 96-well plate. Measurements were obtained at 482/515 nm. Cholesterol efflux was calculated as follows: Cholesterol efflux \% = Fluorescence intensity of media/(Fluorescence intensity of cell lysate + media) $\times 100$.

\section{Real-time polymerase chain reaction}

Real-time polymerase chain reaction (PCR) was performed as previously described [32]. Briefly, total RNA was extracted from cells with Trizol reagent (Invitrogen) and reverse-transcribed with the GoScript Reverse Transcription System (Promega, Madison, WI). Quantitative PCR was performed on the Bio-Rad iQ5 detection system (Bio-Rad, Hercules, CA) with Real-Time PCR Easy (SYBR Green I; FORGENE, Sichuan, China). The following primers were used: ABCA1, forward 5'-TGGCAGTGTCCAGCATCTAA- $3^{\prime}$ and reverse 5'-GTATTGTAGCATGTTCCGGTGT-3'; ABCG1, forward 5'-CGGGGAAAAGTCTGCAAT-3' and reverse 5'-GGTGCCAAAGAAAAGGGT-3'; SR-B1, forward 5'-TCCATCTACCCACCCAACGAG-3' and reverse 5'-GTCAGCGTTGAGGAAGTGAGGAT-3'; CD36, forward 5'-TGAGTTTGGTTCCGTAC

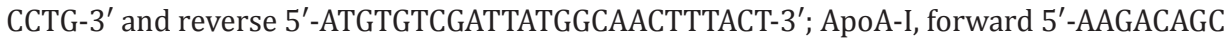

GGCAGAGACTATG-3' and reverse $5^{\prime}$ - CTCTGTCTCCTTTTCCAGGTTAT $-3^{\prime}$; CHOP, forward 5'-TTCACCACTCTTGACCCTGCT-3' and reverse 5'-TCCGTTTCCTGGTTCTCCCTT-3'; miR-33a-5p, forward 5'-CATTGTAGTTGCATTGC-3' and reverse 5'- GGCCAACCGCGAGAAGATG -3'; miR-144a-3p, forward 5'-GCTGGGATATCATCATATACTG-3' and reverse 5'-CGGACTAGTACATCATCTATACTG-3'. Gene expression was normalized to that of GAPDH and U6 snRNA. All samples were performed in duplicate. Data were analyzed using the $2^{-\Delta \Delta C \mathrm{t}}$ method.

\section{Western blot analysis}

Total protein was extracted from frozen crushed aorta tissue and cells by adding ice-cold RIPA buffer containing protease and phosphatase inhibitors. For western blot analysis, equivalent amounts of protein samples were separated on sodium dodecyl sulfate polyacrylamide gel electrophoresis gels and transferred onto polyvinylidene fluoride membranes (Millipore, Schwalbach, Germany), followed by blocking for $2 \mathrm{~h}$ at room temperature with 5\% skim milk (in TBS). Membranes were probed with specific primary antibodies at $4^{\circ} \mathrm{C}$ overnight with slight agitation, then incubated with HRP-conjugated secondary antibodies at room 
temperature for $1 \mathrm{~h}$. Immunoreactivity was visualized by chemiluminescence with the ChemiDoc MP Imaging System (Bio-Rad) and analyzed with ImageJ Lab software (version 4.1, Bio-Rad).

\section{Transfection}

Small interfering RNAs (siRNAs) were used to knock down CHOP and miR-33 in macrophages. An irrelevant 21-nucleotide siRNA was used as negative control (GenePharma, Shanghai, China). The target sequences were as follows: CHOP-sh\#1: 5'-GAGCUCUGAUUGACCGAAUTT-3'; CHOP-sh\#2: 5'-GAAACGGAAACAGAGUGGUTT-3'; CHOP-sh\#3: 5'-GGUCCUGUCUUCAGAUGAATT-3'. The efficiency of CHOP siRNA transfection was confirmed by western blot analysis. The primer used for miR-33 inhibition was 5'-UGCAAUGCAACUACAAUGCAC-3'. Transfection efficiency was detected by real-time PCR.

\section{Statistical analysis}

All data are presented as the mean \pm standard deviation (SD) from at least three independent experiments. Statistical analysis was performed with SAS 9.3 software (SAS Institute Inc., Cary, NC). Differences between groups were detected using Student's t-test or one-way analysis of variance. Statistical significance was defined as $\mathrm{P}<0.05$.

\section{Results}

\section{TUDCA decreases severity of atherosclerosis in ApoE $\%$ mice}

Because ER stress plays an important role in the formation of atherosclerotic plaques, we investigated whether TUDCA, an inhibitor of ER stress, could ameliorate progression of plaque formation in vivo. H\&E-stained cross-sections of the aortic root showed that the lesion area was smaller in the TUDCA group than in the model group (335951 \pm 143395 $\mu \mathrm{m}^{2}$ vs. $639450 \pm 176827 \mu \mathrm{m}^{2}, \mathrm{P}<0.01, \mathrm{n}=6$; Fig. 1a). Further quantification by Oil Red 0 staining demonstrated that the total plaque area (\%) in the aorta was significantly reduced

Fig. 1. TUDCA inhibited atherosclerotic plaque progression in $\mathrm{ApoE}^{-/-}$mice. (a) Representative photomicrographs of aortic root cross-sections stained with $\mathrm{H}$ \& $\mathrm{E}$ in $\mathrm{ApoE}^{-/-}$mice with or without TUDCA treatment after 16 weeks of being fed a highfat diet ( $n=7-8$ mice per group). Left panels: scale bar $=500 \mu \mathrm{m}$; right panels: scale bar = $200 \mu \mathrm{m} .{ }^{* *} \mathrm{P}<0.01$ compared with the model group. (b) Oil Red 0 staining in the aorta and the percentage of en face plaque areas. ${ }^{* *} \mathrm{P}<0.01$ compared with the model group. (c, d) Levels of plasma total cholesterol and LDL-C were quantified in wild-type (WT), model, and TUDCA mice. ${ }^{* * *} \mathrm{P}<0.001$ compared with the WT group; ${ }^{\#} \mathrm{P}<0.05$ compared with the model group. NS, not significant. (e, f) Western blot analysis of ER stress markers GRP78, ATF4, and CHOP and lipid metabolism transporters CD36, ABCA1, and ApoA-I in WT, model, and TUDCA mice. ${ }^{*} \mathrm{P}<0.05$ and ${ }^{* * *} \mathrm{P}<0.001$ compared with the WT group; ${ }^{\#} \mathrm{P}<0.01,{ }^{\# \#} \mathrm{P}<0.01$, and ${ }^{\# \# \#} \mathrm{P}<0.001$ compared with the model group. All values are given as the mean \pm SD (error bars) of three independent experiments.

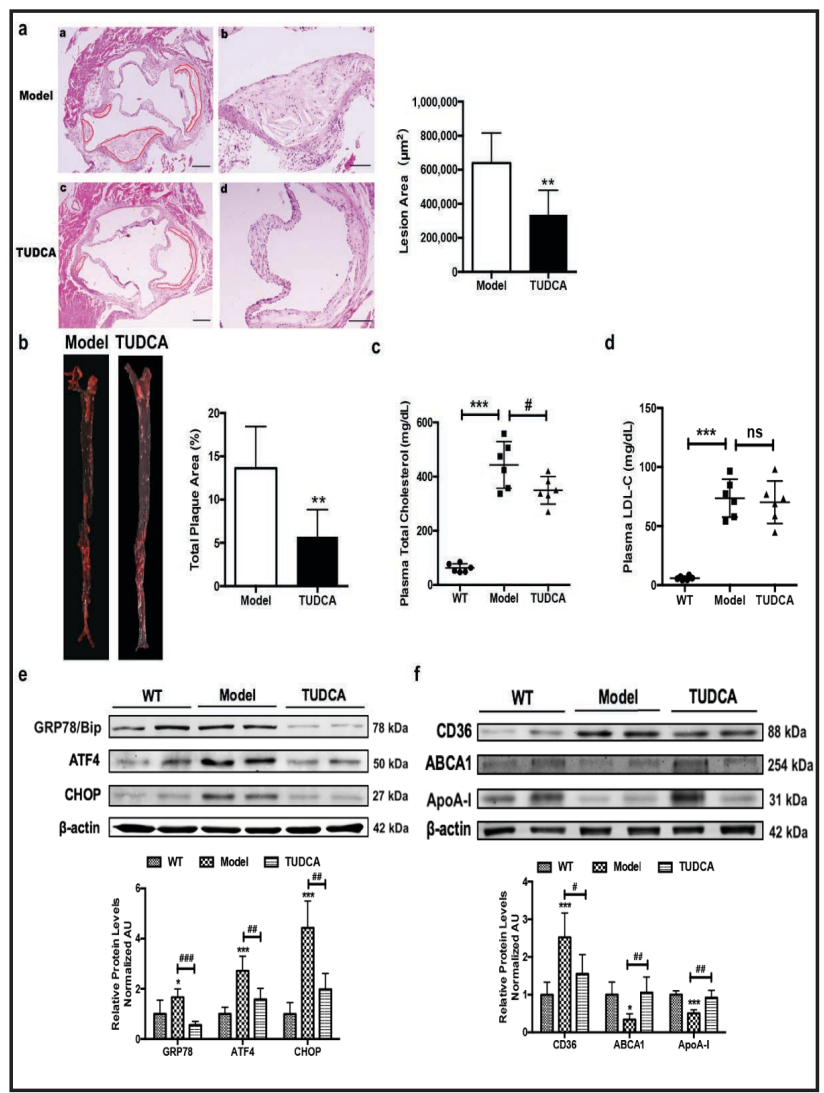


in the TUDCA group compared with the model group $(5.727 \pm 3.106 \%$ vs. $13.63 \pm 4.816 \%, \mathrm{P}<$ $0.01, \mathrm{n}=6$; Fig. 1b), indicating that atherosclerotic plaque formation was inhibited in TUDCA mice. While abnormal lipid metabolism may affect the pathogenesis of atherosclerosis [8], it remains unclear whether TUDCA modulates systemic lipid levels during atherosclerosis. We therefore measured the levels of LDL-C and TC in control, model, and TUDCA mice. The levels of TC and LDL-C were significantly increased in the model and TUDCA group compared with the control group $(\mathrm{P}<0.001, \mathrm{n}=6)$. Plasma $\mathrm{TC}$ content was lower in mice treated with TUDCA compared with the model group $(349.2 \pm 50.88 \mathrm{mg} / \mathrm{dL} v s .443 .0 \pm 86.44 \mathrm{mg} / \mathrm{dL}, \mathrm{P}<$ $0.05, \mathrm{n}=6)$, whereas LDL-C content was comparable between the groups $(70.17 \pm 18.00 \mathrm{mg} /$ dL vs. $73.62 \pm 16.09 \mathrm{mg} / \mathrm{dL}, \mathrm{P}>0.05, \mathrm{n}=6$; Fig. 1c, 1d).

To determine whether TUDCA could inhibit lipid metabolism disorder in atherosclerotic lesions by mitigating ER stress, we analyzed ER stress indicators and lipid catabolism transporters in mouse aorta. Expression of ER stress markers ATF4, CHOP, and GRP78 was increased in model mice compared with controls; this effect was reversed by treatment with TUDCA. In the model group, lipid uptake receptor CD36 was activated, and the levels of cholesterol efflux transporters ABCA1 and ApoA-I decreased. These effects were also reversed by TUDCA treatment (Fig. 1e, 1f). These findings indicate that TUDCA treatment regulates lipid metabolism in lesions and promotes the regression of established atherosclerosis.

\section{Lipid catabolism of macrophage is regulated by ER stress}

To elucidate the functional relevance of lipid catabolism and ER stress, we analyzed the effects of ER stress induction and inhibition (TG and TUDCA) on macrophage lipid uptake and efflux. The results of the DIL-ox-LDL binding assay showed that cholesterol uptake increased by approximately 35\% in TG-treated macrophages compared to control cells. Cholesterol uptake decreased slightly in the TUDCA-treated group (Fig. 2a).

TG significantly reduced cholesterol efflux via TUDCA-promoted macrophage cholesterol efflux (Fig. 2b). Cellular lipid accumulation (as indicated by Oil Red 0 staining) was increased by TG incubation and reduced by pretreatment with TUDCA (Fig. 2c). These findings suggest that the imbalance between lipid uptake and efflux in macrophages is related to ER stress.

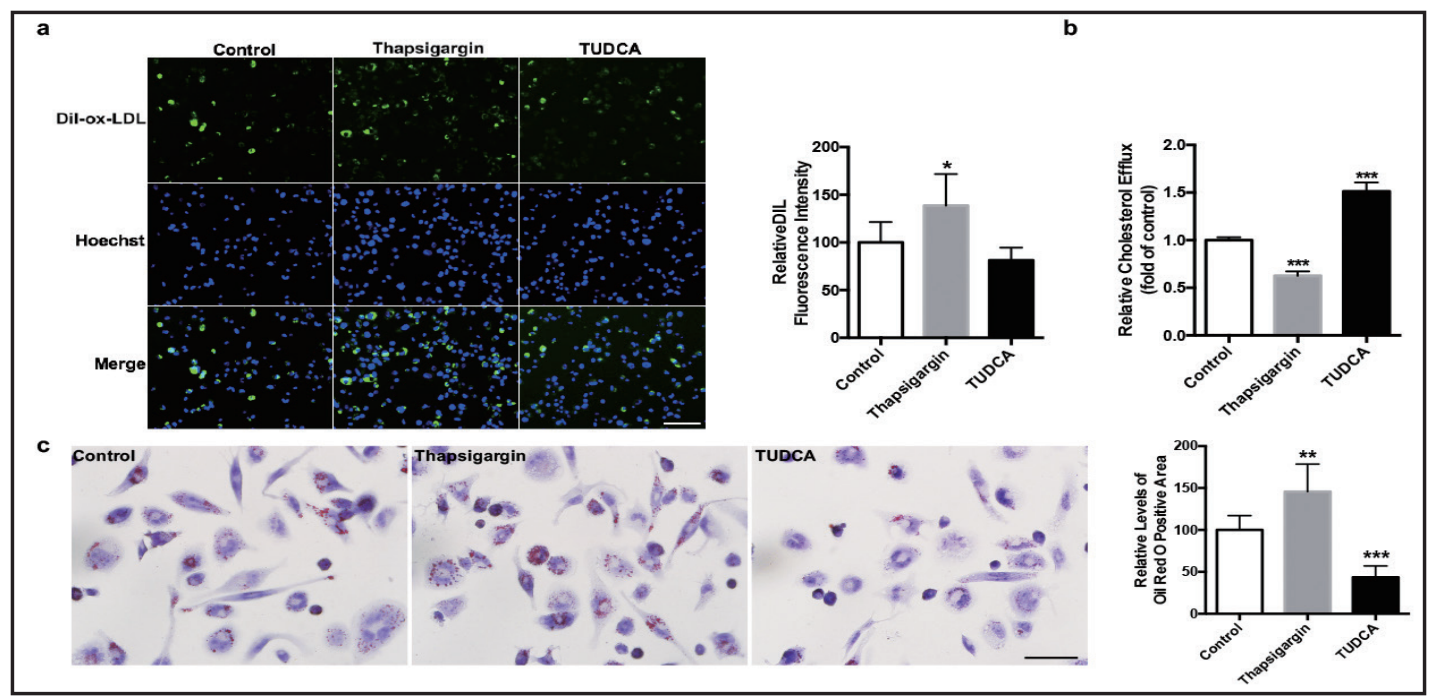

Fig. 2. ER stress regulated lipid catabolism in macrophages. (a) Fluorescence microscopy analysis of the uptake of DIL-ox-LDL in macrophages after TG or TUDCA pre-treatment. Scale bar $=100 \mu \mathrm{m}$. (b) Cholesterol efflux to HDL (50 $\mu \mathrm{g} /$ well) in macrophages after TG or TUDCA treatment for $24 \mathrm{~h}$. (c) Lipid accumulation in macrophages treated with TG or TUDCA for $24 \mathrm{~h}$, as determined by Oil Red 0 staining. Scale bar $=50 \mu \mathrm{m}$. ${ }^{*} \mathrm{P}<0.05,{ }^{* *} \mathrm{P}<0.01$, and ${ }^{* * *} \mathrm{P}<0.001$ compared with the control group. All values are given as the mean $\pm \mathrm{SD}$ (error bars) of three independent experiments. 
ER stress modulates transporters of cholesterol uptake and efflux in macrophages

To further investigate the putative link between ER stress and cholesterol metabolism, we measured the expression of CD36, SR-BI, ABCG1, ABCA1, and ApoA-I, which are transporters essential for cholesterol uptake and efflux in macrophages, after inducing acute ER stress. We applied TG or TM, which impairs protein glycosylation or disturbs intracellular calcium homeostasis and results in ER stress [33, 34]. Macrophages were incubated with TG for 24 h. As expected, TG treatment induced the expression of several UPR markers, including ER chaperone GRP78, ATF4, and CHOP. These effects were dose-dependent (Fig. 3a, 3c). The expression of CD36, an important receptor for cholesterol uptake in macrophages, was

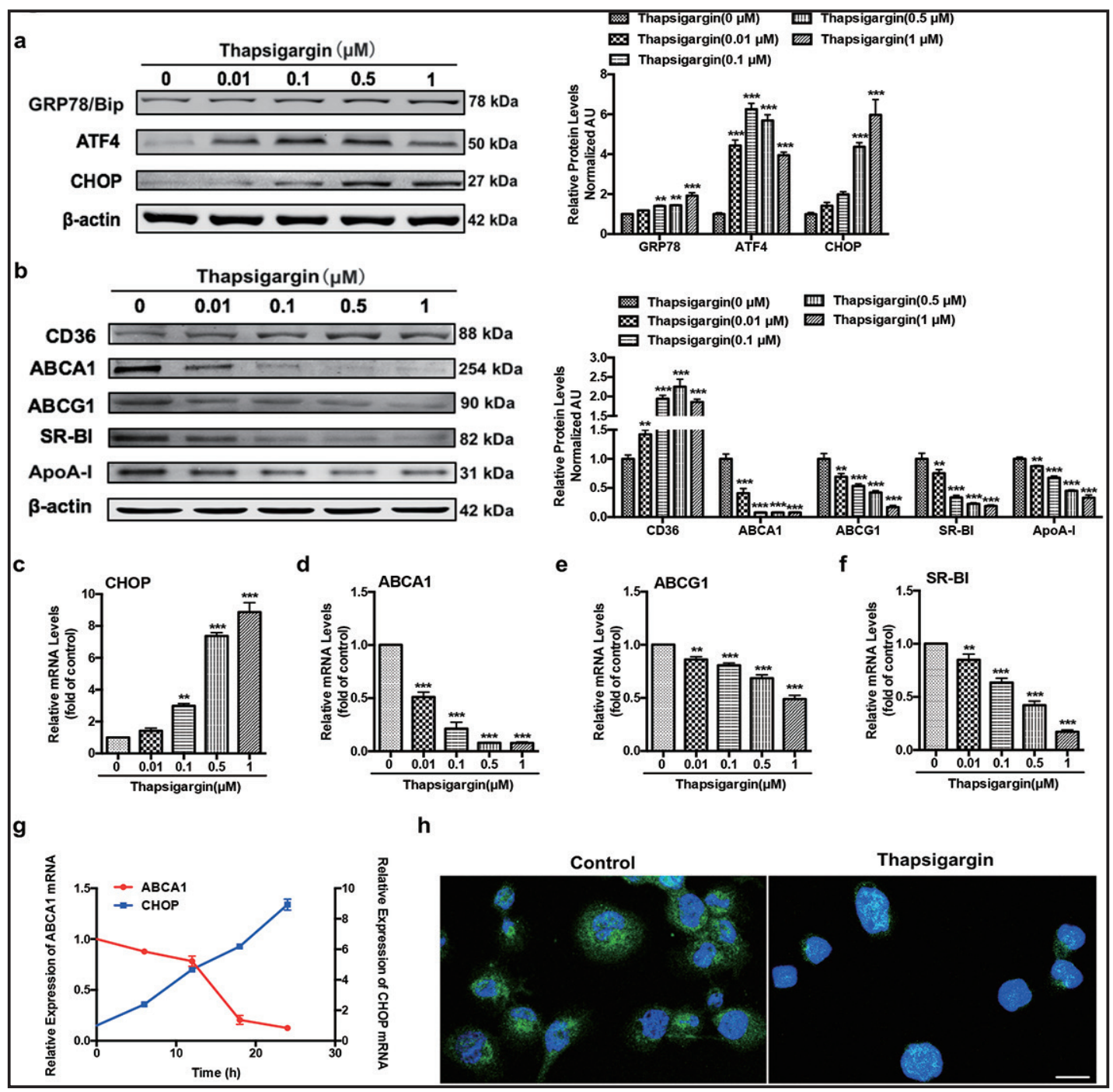

Fig. 3. ER stress regulated the expression of lipid metabolism transporters. Macrophages were incubated with TG for $24 \mathrm{~h}$. (a) Western blot analysis shows expression of ER stress markers GRP78, ATF4, and CHOP. (b) Expression of cholesterol efflux transporters ABCA1, ABCG1, SR-BI, and ApoA-I inhibited by TG treatment, but levels of cholesterol uptake transporter CD36 increased. (c-f) Real time-PCR analysis shows dose-dependent reduction of ABCA1, ABCG1, and SR-BI expression levels after TG treatment, accompanied by increased CHOP levels. (g) ABCA1 and CHOP mRNA expression altered inversely over time after TG treatment $(0.5 \mu \mathrm{M})$. (h) Location of ABCA1 altered to tubular cellular compartments by TG treatment $(0.5 \mu \mathrm{M})$, as visualized by immunofluorescence microscopy. Green: ABCA1; blue: DAPI; scale bar $=25 \mu \mathrm{m}$. ${ }^{* *} \mathrm{P}<0.01$ and ${ }^{* * *} \mathrm{P}<0.001$ compared with control. All values are given as the mean \pm SD (error bars) of three independent experiments. 
increased. Levels of transporters that mediate cholesterol efflux, including ABCA1, ABCG1, SR-BI, and ApoA-I, were reduced at both the transcriptional and translational levels (Fig. 3b, 3d-f). Compared with CHOP expression, ABCA1 mRNA expression exhibited an inverse pattern (Fig. 3g). Furthermore, the location of ABCA1, which is important for its function, was redistributed to tubular perinuclear compartments after TG treatment (Fig. 3h). For further confirmation, macrophages were treated with TM, which independently induced ER stress. Similarly, TM treatment reduced the expression of ABCA1 and ApoA-I, whereas the expression of GRP78, ATF4, CHOP, and CD36 increased (Fig. 4a, 4b). These results suggest a general role for ER stress in the regulation of lipid metabolism in macrophages.

\section{CHOP contributes to macrophage lipid metabolism disorder following ER stress}

CHOP, a protein downstream of the UPR, is induced in advanced murine and human coronary artery plaques [17]. To further support the critical role of CHOP in the regulation of macrophage cholesterol catabolism following ER stress, we measured lipid uptake and efflux in macrophages after treatment with TG (with or without CHOP knockdown via CHOP siRNA). We found that CHOP siRNA \#3 reduced CHOP expression by approximately 59\%, indicating that knockdown was successful $(1.000 \pm 0.05863$ vs. $0.4133 \pm 0.009644, \mathrm{P}<$ $0.001, \mathrm{n}=3$; Fig. 5a). The results of the DIL-ox-LDL binding assay showed that lipid uptake in macrophages, promoted by TG treatment, was significantly reversed by CHOP siRNA (Fig. $5 b)$. The lipid efflux capacity of macrophages was reduced significantly by TG treatment and this effect was effectively reversed by CHOP siRNA (Fig. 5c). Similarly, the results of Oil Red $O$ staining showed that lipid accumulation in macrophages was increased by TG incubation and reduced by CHOP siRNA pretreatment (Fig. 5d). Downstream ER stress signal CHOP appears to be critical for macrophage lipid catabolism in response to alternative activation.

miR-33 contributes to the reduction in ABCA1 levels induced by ER stress

Although we have verified the association between ER stress and ABCA1 reduction, the exact mechanism by which ER stress affects ABCA1 content requires further investigation. miR-33 is an important regulatory factor of lipid homeostasis, which links the sterol regulatory element-binding protein activation to the reduced expression of ABCA1 in humans. Accordingly, we analyzed the expression of miR-33 after ER stress induction in macrophages. As shown in Fig. 6a, miR-33 levels increased significantly after exposure to TG,

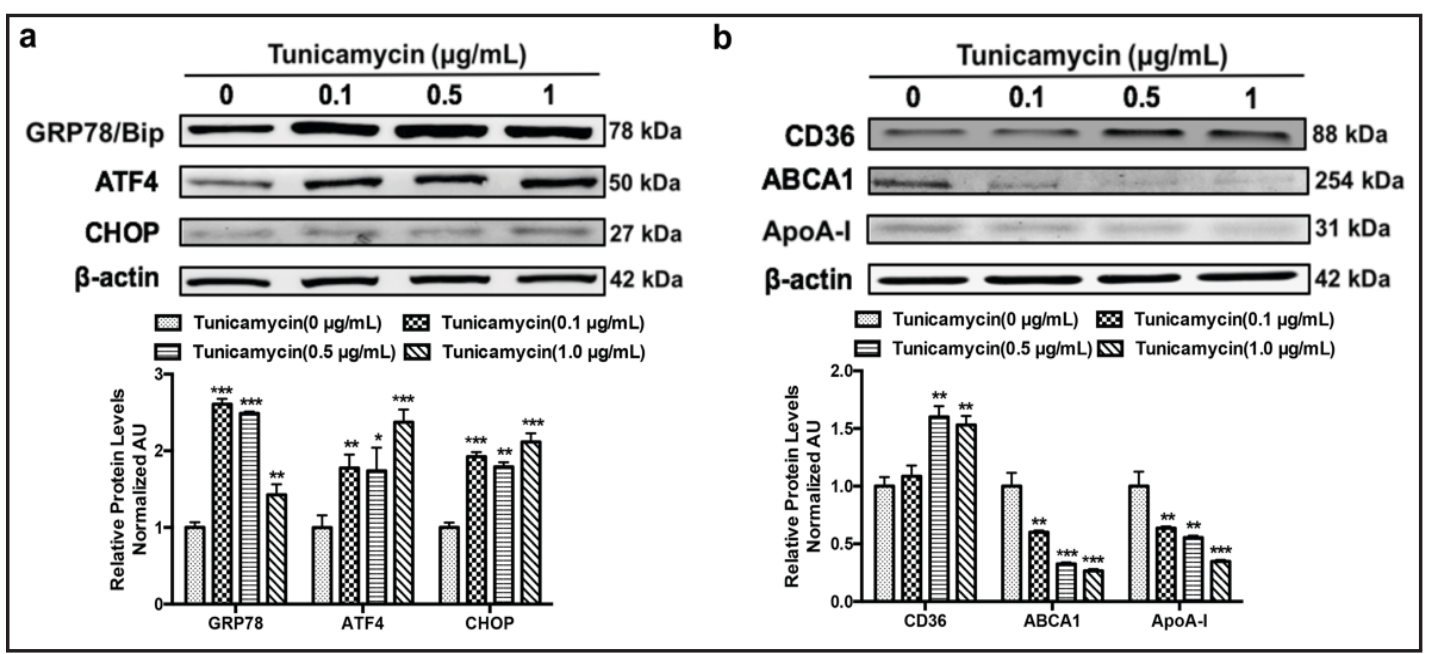

Fig. 4. Induction of ER stress by TM regulates expression of lipid metabolism transporters. Macrophages were incubated with TM for $24 \mathrm{~h}$. (a) Western blot analysis shows expression of ER stress markers GRP78, ATF4, and CHOP. (b) TM treatment reduces expression of ABCA1 and ApoA-I in a dose-dependent fashion, but levels of CD36 are increased. ${ }^{*} \mathrm{P}<0.05,{ }^{* *} \mathrm{P}<0.01$ and ${ }^{* * *} \mathrm{P}<0.001$ compared with control. All values are given as mean \pm S.D. (error bars) of three independent experiments. 


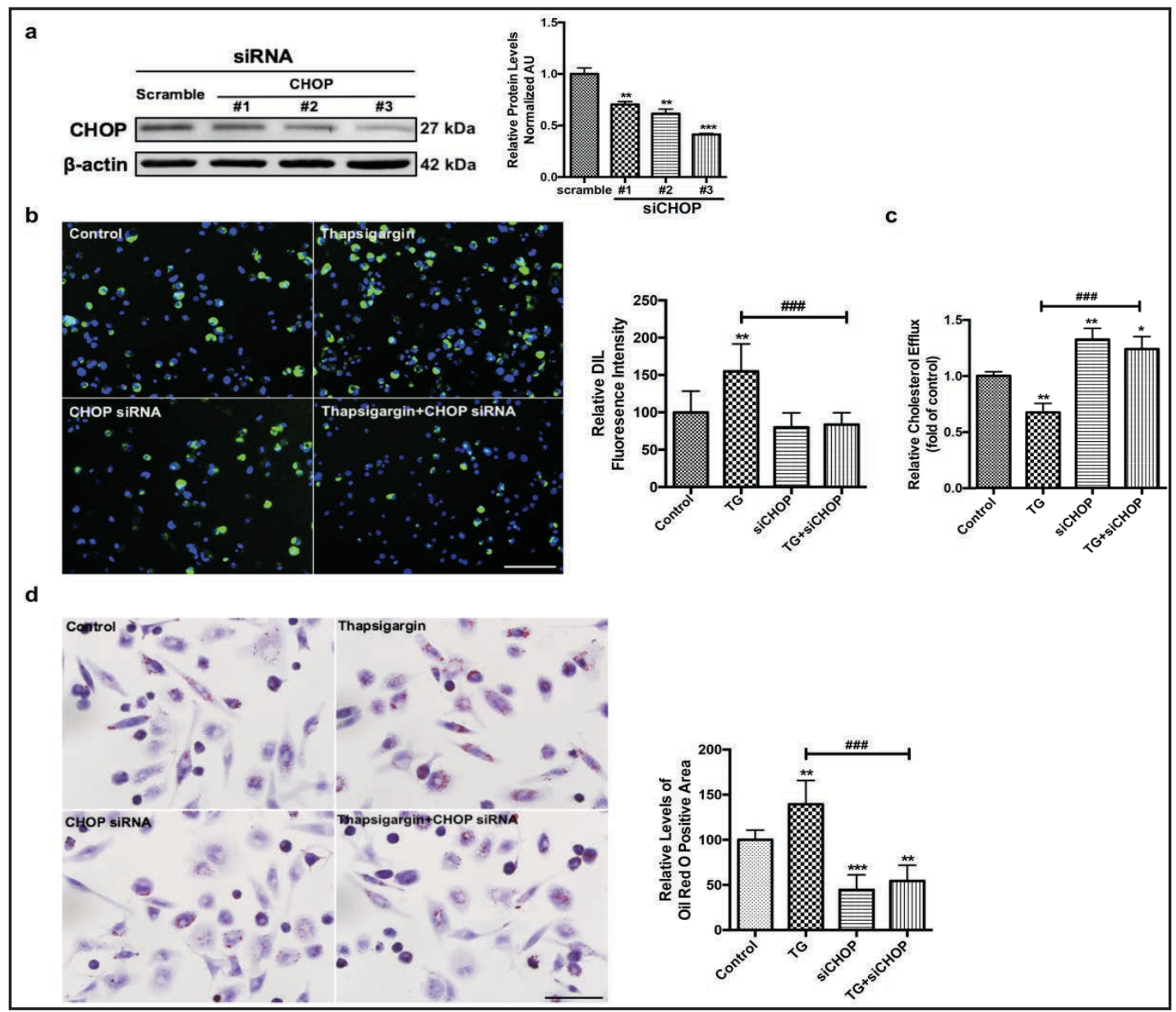

Fig. 5. CHOP was involved in ER stress-induced lipid metabolism disorder. (a) Representative western blots of CHOP following siRNA treatment. (b) Fluorescence microscopy analysis of the uptake of DIL-ox-LDL in macrophages pre-incubated with CHOP siRNA following TG treatment. Scale bar $=100 \mu \mathrm{m}$. (c) Cholesterol efflux to HDL (50 $\mu \mathrm{g} /$ well) in macrophages pre-incubated with CHOP siRNA following TG treatment. (d) Lipid accumulation in macrophages pre-incubated with CHOP siRNA following TG treatment, as determined by Oil Red 0 staining. Scale bar $=50 \mu \mathrm{m} .{ }^{*} \mathrm{P}<0.05,{ }^{* *} \mathrm{P}<0.01$, and ${ }^{* * *} \mathrm{P}<0.001$ compared with control; ${ }^{* \# \#} \mathrm{P}<0.001$ compared with TG. All values are given as the mean \pm SD (error bars) of three independent experiments.

indicating that miR-33 may play a key role in ER stress-induced lipid metabolism disorder in macrophages.

We used siRNA to knock down miR-33 to investigate the effects on lipid efflux (Fig. 6b). As expected, cholesterol efflux was improved in miR-33 knockdown macrophages compared with controls. The reduction in lipid efflux capacity induced by TG treatment was reversed by si-miR-33 (Fig. 6c). After TG incubation, miR-33 knockdown macrophages had accumulated nearly 55\% less cholesterol than normal cells (Fig. 6d). As for the reverse cholesterol transporters closely related to lipid efflux, the decrease in expression of ABCA1 induced by TG treatment was reversed by si-miR-33; knockdown of miR-33 had no significant effect on the protein level of ABCG1, SR-BI, or ApoA-I, which supports our conclusions based on mRNA expression (Fig. 6e-i). These data indicate that miR-33 is involved in the ER stressinduced impairment of lipid efflux capacity and ABCA1 reduction in macrophages.

Ox-LDL influences the expression of ER stress markers and lipid metabolism transporters 


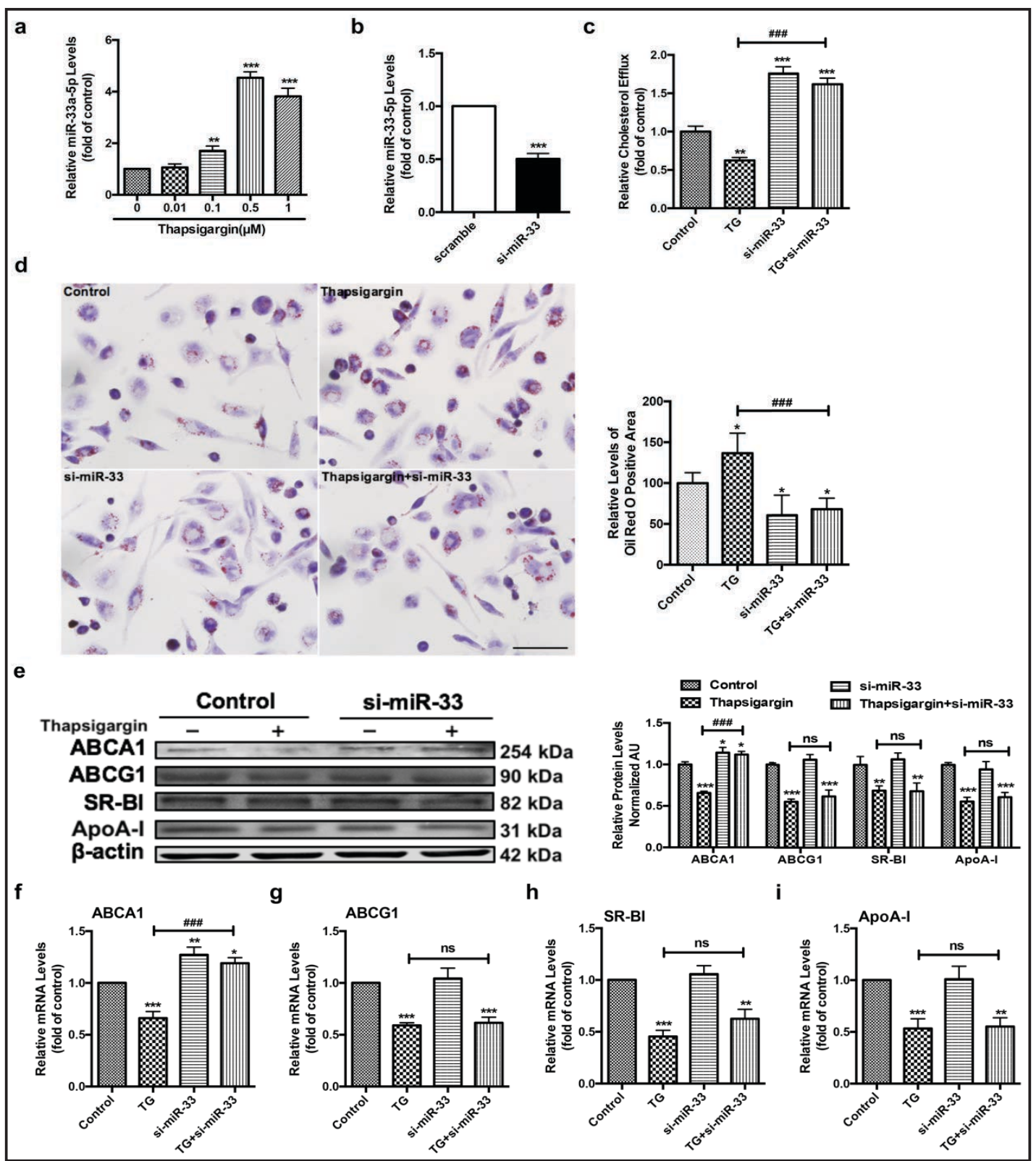

Fig. 6. Alteration of miR-33 expression modulated cholesterol efflux. (a) Expression of miR-33 following TG treatment for 24 h. (b) Real-time PCR analysis of miR-33 in macrophages transfected with negative control or si-miR-33. (c) Cholesterol efflux to HDL (50 $\mu \mathrm{g} /$ well) in macrophages pre-treated with si-miR-33 in the presence or absence of TG. (d) Lipid accumulation in macrophages pre-incubated with si-miR-33 following TG treatment, as determined by Oil Red 0 staining. Scale bar $=50 \mu \mathrm{m}$. (e-i) Western blot and real time-PCR analysis of ABCA1, ABCG1, SR-BI, and ApoA-I in macrophages pre-treated with si-miR-33 in the presence or absence of TG. ${ }^{*} \mathrm{P}<0.05,{ }^{* *} \mathrm{P}<0.01$, and ${ }^{* * *} \mathrm{P}<0.001$ compared with Control; ${ }^{\# \#} \mathrm{P}<0.001$ compared with TG. NS, not significant. All values are given as the mean \pm SD (error bars) of three independent experiments.

\section{in macrophages}

To investigate changes in transporter expression related to lipid homeostasis under ox-LDL-induced ER stress, we measured the activation of ER stress-specific markers in macrophages exposed to ox-LDL. As shown in Fig. 7a and 7c, activation of GRP78, ATF4, and CHOP was induced by ox-LDL in a dose- and time-dependent manner, peaking at $100 \mu \mathrm{g} / \mathrm{mL}$ for $48 \mathrm{~h}$. Concomitantly, the expression of lipid metabolism transporters, including CD36,

\section{KARGER}




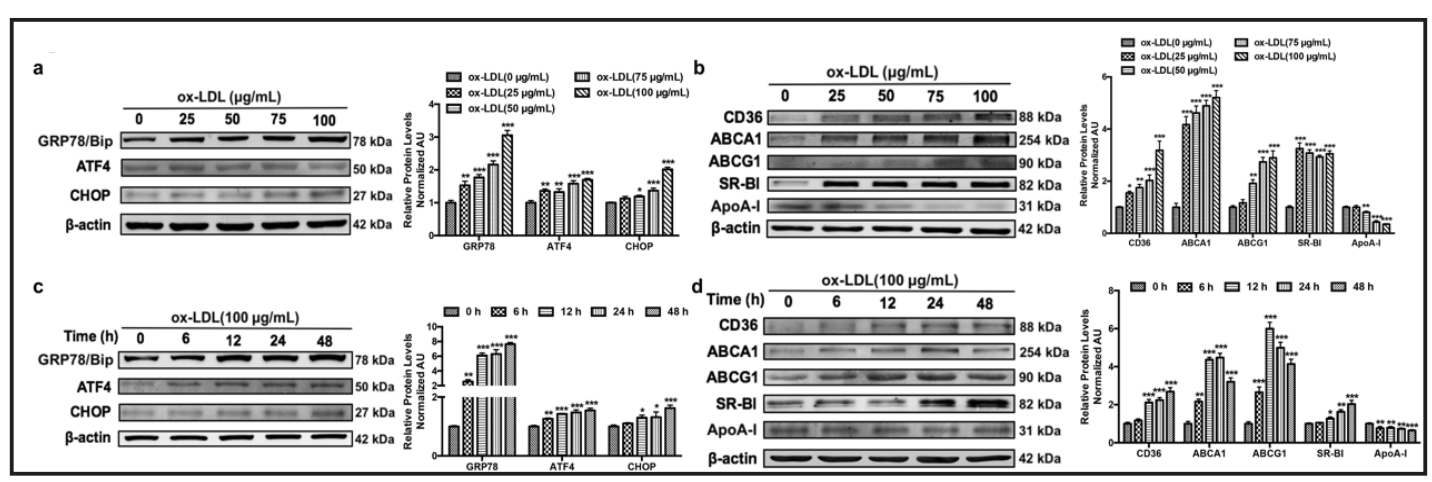

Fig. 7. Ox-LDL influenced the expression of ER stress markers and lipid metabolism transporters in macrophages. (a, c) Western blot analysis of ER stress markers GRP78, ATF4, and CHOP in macrophages after exposure to different doses of ox-LDL at various time points. (b, d) Western blot analysis of cholesterol metabolism transporters CD36, ABCA1, ABCG1, SR-BI, and ApoA-I in macrophages after exposure to different doses of ox-LDL at various time points. ${ }^{*} \mathrm{P}<0.05,{ }^{* *} \mathrm{P}<0.01$, and ${ }^{* * *} \mathrm{P}<0.001$ compared with control. All values are given as the mean \pm SD (error bars) of three independent experiments.

ABCA1, ABCG1, and SR-BI, was increased in cells exposed to ox-LDL compared with controls, indicating that not only ER stress but also other pathways are involved in the lipid metabolism disorder induced by ox-LDL in macrophages. Moreover, the expression of ApoA-I, another primary transporter of lipid efflux, was significantly reduced by ox-LDL (Fig. 7b, 7d). We then assessed the relationship between mRNA expression of CHOP and ABCA1 after exposure to oxLDL $(100 \mu \mathrm{g} / \mathrm{mL})$. The ABCA1 mRNA level peaked $18 \mathrm{~h}$ after ox-LDL treatment, while as CHOP mRNA levels increased, ABCA1 levels decreased. ABCA1 levels were lowest at $72 \mathrm{~h}$, indicating that cholesterol efflux was reduced under prolonged ox-LDL-

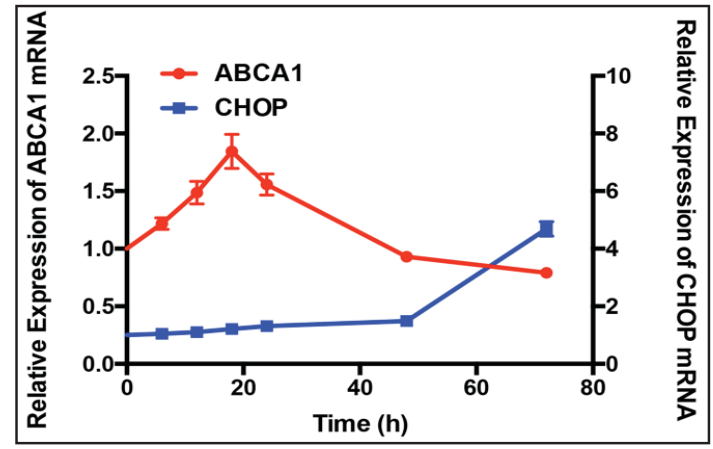

Fig. 8. Relationship between mRNA expression of CHOP and ABCA1 after exposure to ox-LDL. ABCA1 mRNA level peaked $18 \mathrm{~h}$ after ox-LDL treatment $(100$ $\mu \mathrm{g} / \mathrm{mL}$ ). As CHOP mRNA levels increased, ABCA1 levels declined (nadir at $72 \mathrm{~h}$ ). induced ER stress (Fig. 8).

\section{ER stress contributes to ox-LDL-induced lipid metabolism disorder in macrophages}

To further clarify whether ER stress is responsible for ox-LDL-induced lipid metabolism disorder in macrophages, we treated macrophages with or without TUDCA, during ox-LDL incubation. TUDCA decreased ox-LDL-induced ER stress, as demonstrated by reduced UPR protein activation compared with that observed in non-TUDCA treated cells (Fig. 9a). TUDCA treatment decreased the expression of CD36 induced by ox-LDL and further increased the levels of ABCA1, ABCG1, SR-BI, and ApoA-I (Fig. 9b). Furthermore, we investigated whether CHOP and miR-33 were involved in ox-LDL-induced lipid metabolism disorder. As shown in Fig. 9c, the increase in CD36 levels induced by ox-LDL was reversed by CHOP siRNA. Transporters involved in cholesterol efflux were further activated in macrophages pretreated with CHOP siRNA compared with macrophages that underwent ox-LDL incubation alone. simiR-33 contributed only to ABCA1 levels and had no impact on other transporters. 
Fig. 9. Effect of ER stress on ox-LDLinduced lipid metabolism disorder in macrophages. (a) Western blot analysis of ER stress markers GRP78, ATF4, and CHOP in macrophages pre-incubated with TUDCA following ox-LDL $(100 \mu \mathrm{g} / \mathrm{mL})$ treatment for 48 h. (b) Western blot analysis of cholesterol metabolism transporters CD36, ABCA1, ABCG1, SR-BI, and ApoA-I in macrophages preincubated with TUDCA following ox-LDL $(100 \mu \mathrm{g} / \mathrm{mL})$ treatment for 48 h. (c) Western blot analysis of cholesterol metabolism transporters CD36, ABCA1, ABCG1, SR-BI,

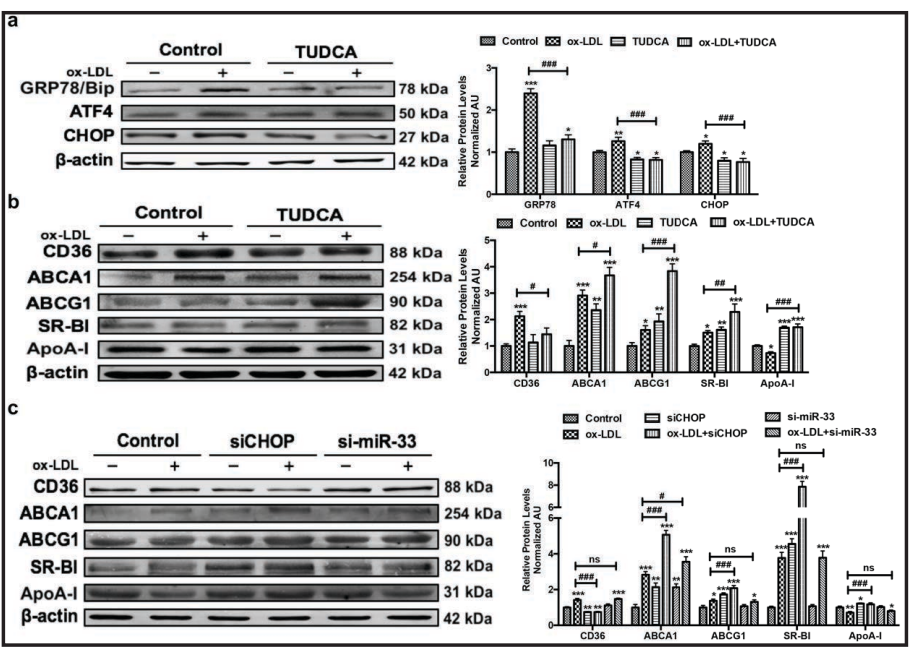
and ApoA-I in macrophages pre-

incubated with CHOP siRNA or miR-33 siRNA following ox-LDL $(100 \mu \mathrm{g} / \mathrm{mL})$ treatment for $48 \mathrm{~h} .{ }^{*} \mathrm{P}<0.05$, ${ }^{* *} \mathrm{P}<0.01$, and ${ }^{* * *} \mathrm{P}<0.001$ compared with control; ${ }^{\# \#} \mathrm{P}<0.01$ and ${ }^{\# \# \#} \mathrm{P}<0.001$ compared with ox-LDL. NS, not significant. All values are given as the mean \pm SD (error bars) of three independent experiments.

\section{Discussion}

This study provides new evidence showing that atherosclerotic lesions and systemic lipid levels in ApoE $\%$ mice are ameliorated following TUDCA treatment. Furthermore, overexpression of miR-33 was involved in the ER stress that induced lipid metabolism disorder in macrophages. Our findings also support a model of ER stress induction by ox-LDL that affects macrophage lipid catabolism, suggesting that ER stress is a potential therapeutic target for the treatment of atherosclerosis (Fig. 10).

ER stress can adversely affect cell biological processes and exacerbate systemic risk factors for atherosclerosis [11]. Human atherosclerotic coronary artery lesions showed a marked increase in GRP78, glucose-regulated protein 94, and CHOP expression in the thin-cap fibroatheroma or ruptured plaques, which suggests that ER stress may affect the vulnerability of plaques as well as the acute complications of coronary atherosclerosis [18]. Zhou et al [14]. showed that activation of the UPR occurs at all stages of atherosclerotic lesion development in ApoE ${ }^{-/}$mice. Moreover, the administration of phenyl butyric acid, an ER stress inhibitor, reduces lipid-induced macrophage apoptosis and overall vascular lesion burden in vitro and in vivo [35]. Similarly, we found that atherosclerosis lesions and systemic lipid levels were ameliorated by inhibition of ER stress in ApoE $\mathrm{E}^{-/}$mice, and this was accompanied by a reversal in the expression of lipid catabolism transporters. These observations indicate that ER function affects overall vascular lesion development and suggest that ER stress not only contributes to cell death but also plays a broader role in the pathophysiological process of atherosclerosis.

Lipid metabolism has a direct association with ER stress because many key lipogenic pathways are situated in the ER [36]. Existing data support the view that ER stress promotes lipogenesis and lipid accumulation in hepatic cells [37,38]. Macrophage studies typically focus on the major role of UPR activation in advanced lesional macrophage death and plaque necrosis, but few studies have investigated lipid metabolism in macrophages [11]. Yao et al [39]. reported that ER stress promotes lipid accumulation in macrophages by upregulating CD36 expression. We further demonstrated that lipid catabolism and related transporters in macrophages are regulated by ER stress. Moreover, the downstream ER stress signal (CHOP activation) and miR-33 over-expression are critical to this process. Hence, modulation of the upstream pathways of apoptosis induced by ER stress may provide new insights into the pathogenesis of atherosclerosis and the associated effects on macrophage lipid metabolism. 


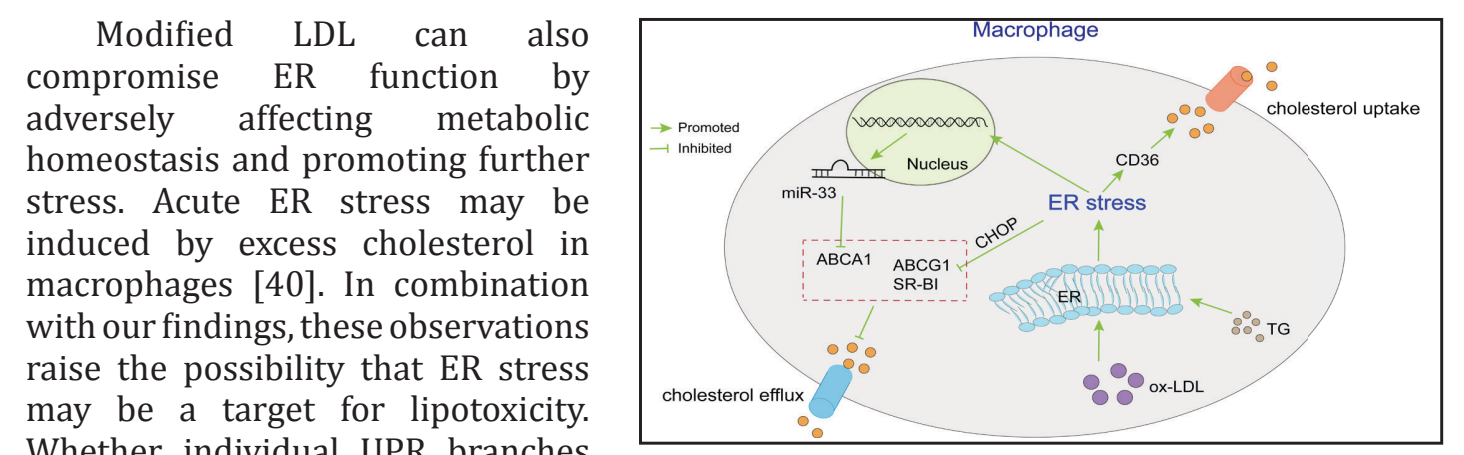
Whether individual UPR branches become engaged remains unclear. Moreover, levels of cholesterol efflux transporters including ABCA1, ABCG1, and SR-BI were increased by ox-LDL in our study, which is in accordance with previous findings [41, 42]. This difference between TG and ox-LDL treatment reveals that not only ER stress but also other pathways are involved in lipid metabolism disorder induced by ox-LDL in macrophages. Lipidinduced ER stress and cholesterol metabolism disorder in cultured macrophages were also mitigated by treatment with TUDCA or CHOP knockdown, which suggests a potential therapeutic target for macrophage lipid disorder in atherosclerosis. To further explore the pathophysiological process of disease and reveal the unknown aspects of ER biology and the UPR pathway, we sought to elucidate the mechanisms involved in lipidinduced ER stress.

As hypercholesterolemia has repeatedly been associated with atherosclerosis, strict regulation of lipid metabolism is essential for the maintenance of cholesterol homeostasis. Over recent decades, miRNAs have been proposed as central regulators of lipid and lipoprotein metabolism [43-45]. The miR-33 family has attracted attention because of its role in the regulation of genes involved in HDL metabolism, cholesterol efflux, fatty acid oxidation, and bile acid synthesis and secretion [46]. Several studies have shown that miR-33 regulates cholesterol efflux by targeting the ABCA1cholesterol transporter gene in a variety of cell types $[23,47]$. We found that ER stress decreases ABCA1 levels in human macrophages via over-expression of miR-33, which suggests that it has an important role in ER stress-induced cholesterol metabolism disorder. However, the level of miR-144, which is reported to directly target ABCA1 in mouse and human hepatocytes in order to decrease cholesterol levels [25, 48, 49], was unaltered in macrophages after exposure to TG (Fig. 11).

In summary, the findings of this study shed new light on how ER stress leads to lipid metabolism disorder in vivo and in vitro. Challenging but crucial problems related to the 


\section{Cellular Physiology Cell Physiol Biochem 2018;48:1995-2010 \begin{tabular}{l|l|l} 
DOI: 10.1159/000492522 & 2018 The Author(s). Published by S. Karger AG, Basel
\end{tabular} and BiOchemistry Published online: August 8, 2018 www.karger.com/cpb}

metabolic regulation of ER function and UPR in atherosclerosis may be solved through the development of superior chemical and genetic tools.

\section{Acknowledgements}

This work was supported by the National Key Research and Development Program of China (2017YFC0908800), the National Natural Science Foundation of China (81100198), Beijing Municipal Administration of Hospitals Ascent Plan (DFL20150601), the National Key Clinical Specialty Construction Project, and Beijing Key Laboratory of Precision Medicine of Coronary Atherosclerotic Disease.

\section{Disclosure Statement}

The authors declare no conflicts of interest.

\section{References}

1 Hansson GK, Libby P: The immune response in atherosclerosis: a double-edged sword. Nat Rev Immunol 2006;6:508-519.

2 Moore K, Sheedy F, Fisher E: Macrophages in atherosclerosis: a dynamic balance. Nat Rev Immunol 2013;13:709-721.

-3 Moore KJ, Tabas I: Macrophages in the pathogenesis of atherosclerosis. Cell 2011;145:341-355.

4 Kunjathoor VV, Febbraio M, Podrez EA, Moore KJ, Andersson L, Koehn S, Rhee JS, Silverstein R, Hoff HF, Freeman MW: Scavenger receptors class A-I/II and CD36 are the principal receptors responsible for the uptake of modified low density lipoprotein leading to lipid loading in macrophages. J Biol Chem 2002;277:49982-49988.

5 Rader DJ, Tall AR: The not-so-simple HDL story: Is it time to revise the HDL cholesterol hypothesis? Nat Med 2012;18:1344-1346.

6 Kleemann R, Zadelaar S, Kooistra T: Cytokines and atherosclerosis: a comprehensive review of studies in mice. Cardiovasc Res 2008;79:360-376.

7 Lusis AJ: Atherosclerosis. Nature 2000;407:233-241.

8 Libby P, Ridker PM, Hansson GK: Progress and challenges in translating the biology of atherosclerosis. Nature 2011;473:317-325.

-9 Liu M, Chen Z, Chen L: Endoplasmic reticulum stress: a novel mechanism and therapeutic target for cardiovascular diseases. Acta Pharmacol Sin 2016;37:425-443.

10 Ron D, Walter P: Signal integration in the endoplasmic reticulum unfolded protein response. Nat Rev Mol Cell Biol 2007;8:519-529.

11 Tabas I: The role of endoplasmic reticulum stress in the progression of atherosclerosis. Circ Res 2010;107:839-850.

$\checkmark 12$ Cominacini L, Garbin U, Mozzini C, Stranieri C, Pasini A, Solani E, Tinelli IA, Pasini AF: The atherosclerotic plaque vulnerability: focus on the oxidative and endoplasmic reticulum stress in orchestrating the macrophage apoptosis in the formation of the necrotic core. Curr Med Chem 2015;22:1565-1572.

13 Myoishi M, Hao H, Minamino T, Watanabe K, Nishihira K, Hatakeyama K, Asada Y, Okada K, Ishibashi-Ueda H, Gabbiani G, Bochaton-Piallat ML, Mochizuki N, Kitakaze M: Increased endoplasmic reticulum stress in atherosclerotic plaques associated with acute coronary syndrome. Circulation 2007;116:1226-1233.

14 Zhou J, Lhoták Š, Hilditch BA, Austin RC: Activation of the unfolded protein response occurs at all stages of atherosclerotic lesion development in apolipoprotein E-deficient mice. Circulation 2005;111:1814-1821.

15 Garbin U, Stranieri C, Pasini A, Baggio E, Lipari G, Solani E, Mozzini C, Vallerio P, Cominacini L, Fratta Pasini AM: Do oxidized polyunsaturated Fatty acids affect endoplasmic reticulum stress-induced apoptosis in 


\section{Cellular Physiology Cell Physiol Biochem 2018;48:1995-2010 \begin{tabular}{ll|l} 
and Biochemistry Published online: August 8, 2018 & $\begin{array}{l}\text { (c) } 2018 \text { The Author(s). Published by S. Karger AG, Basel } \\
\text { www.karger.com/cpb }\end{array}$
\end{tabular}}

human carotid plaques? Antioxid Redox Signal 2014;21:850-858.

16 Röhrl C, Eigner K, Winter K, Korbelius M, Obrowsky S, Kratky D, Kovacs WJ, Stangl H: Endoplasmic reticulum stress impairs cholesterol efflux and synthesis in hepatic cells. J Lipid Res 2014;55:94-103.

17 Ivanova EA, Orekhov AN: The role of endoplasmic reticulum stress and unfolded protein response in atherosclerosis. Int J Mol Sci 2016;17:193.

18 Myoishi M, Hao H, Kitakaze M: Increased endoplasmic reticulum stress in atherosclerotic plaques associated with acute coronary syndromes. Circulation 2006;41:1214-1216.

19 Zhang T, Hu Q, Shi L, Qin L, Zhang Q, Mi M: Equol Attenuates Atherosclerosis in Apolipoprotein E-Deficient Mice by Inhibiting Endoplasmic Reticulum Stress via Activation of Nrf2 in Endothelial Cells. PloS One 2016;11: e0167020.

20 Yao S, Zong C, Zhang Y, Sang H, Yang M, Jiao P, Fang Y, Yang N, Song G, Qin S: Activating transcription factor 6 mediates oxidized LDL-induced cholesterol accumulation and apoptosis in macrophages by up-regulating CHOP expression. J Atheroscler Thromb 2013;20:94-107.

-21 Andreou I, Sun X, Stone PH, Edelman ER, Feinberg MW: miRNAs in atherosclerotic plaque initiation, progression, and rupture. Trends Mol Med 2015;21:307-318.

-22 Bartel DP: MicroRNAs: target recognition and regulatory functions. Cell 2009;136:215-233.

23 Rayner KJ, Suárez Y, Dávalos A, Parathath S, Fitzgerald ML, Tamehiro N, Fisher EA, Moore KJ, FernándezHernando C: MiR-33 contributes to the regulation of cholesterol homeostasis. Science 2010;328:15701573.

-24 Najafi-Shoushtari SH, Kristo F, Li Y, Shioda T, Cohen DE, Gerszten RE, Näär AM: MicroRNA-33 and the SREBP host genes cooperate to control cholesterol homeostasis. Science 2010;328:1566-1569.

25 Karunakaran D, Rayner KJ: Macrophage miRNAs in atherosclerosis. Biochim Biophys Acta 2016;1861:2087-2093.

-26 Rayner KJ, Sheedy FJ, Esau CC, Hussain FN, Temel RE, Parathath S, van Gils JM, Rayner AJ, Chang AN, Suarez Y, Fernandez-Hernando C, Fisher EA, Moore KJ: Antagonism of miR-33 in mice promotes reverse cholesterol transport and regression of atherosclerosis. J Clin Invest 2011;121:2921-2931.

27 Ouimet M, Ediriweera HN, Gundra UM, Sheedy FJ, Ramkhelawon B, Hutchison SB, Rinehold K, van Solingen C, Fullerton MD, Cecchini K, Rayner KJ, Steinberg GR, Zamore PD, Fisher EA, Loke P, Moore KJ: MicroRNA-33-dependent regulation of macrophage metabolism directs immune cell polarization in atherosclerosis. J Clin Invest 2015;125:4334-4348.

28 Horie T, Baba O, Kuwabara Y, Chujo Y, Watanabe S, Kinoshita M, Horiguchi M, Nakamura T, Chonabayashi K, Hishizawa M, Hasegawa K, Kume N, Yokode M, Kita T, Kimura T, Ono K: MicroRNA-33 deficiency reduces the progression of atherosclerotic plaque in ApoE-/- mice. J Am Heart Assoc 2012;1:e003376.

-29 Karunakaran D, Thrush AB, Nguyen M-A, Richards L, Geoffrion M, Singaravelu R, Ramphos E, Shangari P, Ouimet M, Pezacki JP, Moore KJ, Perisic L, Maegdefessel L, Hedin U, Harper ME, Rayner KJ: Macrophage mitochondrial energy status regulates cholesterol efflux and is enhanced by anti-miR33 in atherosclerosis. Circ Res 2015;117:266-278.

-30 Mandolini C, Santovito D, Marcantonio P, Buttitta F, Bucci M, Ucchino S, Mezzetti A, Cipollone F: Identification of microRNAs 758 and 33b as potential modulators of ABCA1 expression in human atherosclerotic plaques. Nutr Metab Cardiovasc Dis 2015;25:202-209.

-31 Craige SM, Kant S, Reif M, Chen K, Pei Y, Angoff R, Sugamura K3, Fitzgibbons T, Keaney JF Jr: Endothelial NADPH oxidase 4 protects ApoE-/- mice from atherosclerotic lesions. Free Radic Biol Med 2015;89:1-7.

-32 Sun Y, Peng PA, Ma Y, Liu XL, Yu Y, Jia S, Xu XH, Wu SJ, Zhou YJ: Valsartan Protects Against Contrast-Induced Acute Kidney Injury in Rats by Inhibiting Endoplasmic Reticulum Stress-Induced Apoptosis. Curr Vasc Pharmacol 2017;15:174-183.

-33 Booth C, Koch GL: Perturbation of cellular calcium induces secretion of luminal ER proteins. Cell 1989;59:729-737.

34 Dorner AJ, Wasley LC, Raney P, Haugejorden S, Green M, Kaufman RJ: The stress response in Chinese hamster ovary cells. Regulation of ERp72 and protein disulfide isomerase expression and secretion. J Biol Chem 1990;265:22029-22034.

35 Erbay E, Babaev VR, Mayers JR, Makowski L, Charles KN, Snitow ME, Fazio S, Wiest MM, Watkins SM, Linton MF, Hotamisligil GS: Reducing endoplasmic reticulum stress through a macrophage lipid chaperone 


\section{Cellular Physiology Cell Physiol Biochem 2018;48:1995-2010 \begin{tabular}{l|l} 
DOI: 10.1159/000492522 \\
and Biochemistry Published online: August 8, 2018 & $\begin{array}{l}\text { O 2018 The Author(s). Published by S. Karger AG, Basel } \\
\text { www.karger.com/cpb }\end{array}$ \\
\hline
\end{tabular}}

alleviates atherosclerosis. Nat Med 2009;15:1383-1391.

-36 Gregor MF, Hotamisligil GS: Thematic review series: Adipocyte Biology. Adipocyte stress: the endoplasmic reticulum and metabolic disease. J Lipid Res 2007;48:1905-1914.

-37 Rutkowski DT, Wu J, Back SH, Callaghan MU, Ferris SP, Iqbal J, Clark R, Miao H, Hassler JR, Fornek J, Katze MG, Hussain MM, Song B, Swathirajan J, Wang J, Yau GD, Kaufman RJ: UPR pathways combine to prevent hepatic steatosis caused by ER stress-mediated suppression of transcriptional master regulators. Dev Cell 2008;15:829-840.

-38 Lee AH, Scapa EF, Cohen DE, Glimcher LH: Regulation of Hepatic Lipogenesis by the Transcription Factor XBP1. Science 2008;320:1492-1496.

39 Yao S, Miao C, Tian H, Sang H, Yang N, Jiao P, Han J, Zong C, Qin S: Endoplasmic Reticulum Stress Promotes Macrophage-derived Foam Cell Formation by Up-regulating Cluster of Differentiation 36 (CD36) Expression. J Biol Chem 2014;289:4032-4042.

40 Tabas I: Macrophage apoptosis in atherosclerosis: consequences on plaque progression and the role of endoplasmic reticulum stress. Antioxid Redox Signal 2009;11:2333-2339.

41 Xian-Ming S, Yang W, Ying W, Wei Z: ABCA1 mRNA expression and cholesterol outflow in U937 cells. Int J Clin Exp Pathol 2015;8:3116-3121.

42 Ledda A, Gonzalez M, Gulfo J, Ludovico ID, Ramella N, Toledo J, Garda H2, Grasa M1, Esteve M: Decreased OxLDL uptake and cholesterol efflux in THP1 cells elicited by cortisol and by cortisone through 11ß-hydroxysteroid dehydrogenase type 1. Atherosclerosis 2016;250:84-94.

43 Desgagné V, Bouchard L, Guérin R: microRNAs in lipoprotein and lipid metabolism: from biological function to clinical application. Clin Chem Lab Med 2016;55:667-686.

44 Fernándezhernando C, Ramírez CM, Goedeke L, Suárez Y: MicroRNAs in Metabolic Disease. Arterioscler Thromb Vasc Biol 2013;33:178-185.

\5 Fernándezhernando C, Suárez Y, Rayner KJ, Moore KJ: MicroRNAs in lipid metabolism. Curr Opin Lipidol 2011;22:86-92.

46 Sahebkar A, Watts GF: Developing role of microRNA-33 in lipid metabolism and atherosclerosis. Curr Opin Lipidol 2016;27:197-199.

47 Fernández-Hernando C, Moore KJ: MicroRNA modulation of cholesterol homeostasis. Arterioscler Thromb Vasc Biol 2011;31:2378-2382.

-48 de Aguiar Vallim TQ Tarling EJ, Kim T, Civelek M, Baldán Á, Esau C, Edwards PA: MicroRNA-144 regulates hepatic ATP binding cassette transporter A1 and plasma high-density lipoprotein after activation of the nuclear receptor farnesoid X receptor. Circ Res 2013;112:1602-1612.

49 Ramírez CM, Rotllan N, Vlassov AV, Dávalos A, Li M, Goedeke L, Aranda JF, Cirera-Salinas D, Araldi E, Salerno A, Wanschel A, Zavadil J, Castrillo A, Kim J, Suárez Y, Fernández-Hernando C: Control of cholesterol metabolism and plasma high-density lipoprotein levels by microRNA-144. Circ Res 2013;112:1592-1601. 